\title{
Infant hearing screening with the combination of otoacoustic emissions and automated auditory brainstem response test : Is it worth it?
}

\author{
Z.Zachou, A.Leventi, A.Katsi, K.Parpounas, I.Athanasopoulos
}

\section{Introduction}

Infant hearing loss is the most common congenital sensory disorder with over 665.000 babies being born annually with significant hearing loss.

The advent of technology improvements in assessing the hearing of newborn infants has made possible the implementation of universal newborn hearing screening (1) and this could lead to the accurate and rapid diagnose of children with permanent hearing loss. An accurate and timely diagnosis is necessary for appropriate amplification, planning of early intervention services and future education options.(3)

The methods that are used most widely are evoked otoacoustic emissions (OAE's) and automated auditory brainstem response (AABR) either separately or in combination.

\section{Materials and Methods}

For a period of 18 months, 469 infants underwent hearing screening tests in our department. The protocol used was history, clinical examination and tympanometry followed by further examination with OAE's and AABR for each infant having risk factors or not. Healthy newborns with normal results (OAE's \& AABR pass) did not need further follow up. Newborns having at least one risk factor with good results were tested every 6 months with OAE's until the age of 3 .

\section{Results}

Of the infants examined, 34 were considered healthy "well" babies without any complicating medical problems and 435 had at least one risk factor. The commonest risk factor that lead the infants to screening test was prematurity.

From the 469 babies that were examined 437 passed the AABR test having a normal follow up, while 2 out of 469 appeared to be false positive, which due to repetitive negative OAEs in follow up and high suspicion from the history were lead to ABR \& ASSR exams which proved a hearing loss (one light and one severe).

\section{Bibliography}

1.Pediatrics. 2005 Oct;116(4):933-8.Newborn hearing screening in the NICU: profile of failed auditory brainstem response/passed otoacoustic emission.Berg AL1, Spitzer JB, Towers HM, Bartosiewicz C, Diamond BE.

2. Int J Pediatr Otorhinolaryngol. 2008 Oct;72(10):1461-9. doi:

10.1016/j.ijporl.2008.06.010. Epub 2008 Jul 31.The frequency of auditory neuropathy detected by universal newborn hearing screening program.Kirkim G1, Serbetcioglu B, Erdag TK, Ceryan K.

3. The sensitivity and specificity of automated auditory brainstem response and otoacoustic emission in neonatal hearing screening: a systematic review.Saeed Heidari, Alireza Olyaee Manesh, Fatemeh Rajabi. Auditory and Vestibular Research 2015. 24(3):141-151.
From the 437 pass AABR 369 had a match with OAE's (positive). In 76 infants OAE's were false negative and from these more than half were negative due to pathology in the middle ear. In our sample none of OAE's was false positive whereas 24 were true negative.

From the 30 refer AABR 12 were unilateral and 18 bilateral. There were 5 out of 30 which did not came for further examination, 8 that due to normal OAE's and history was decided to have a new AABR which appeared normal and in $\mathbf{1 7}$ we had further examination with $A B R$ and ASSR exams which revealed a hearing loss in $\mathbf{1 5}$ infants and a normal hearing in $\mathbf{3}$. More details about the level of hearing loss and the separated unilateral and bilateral results are shown in Table 1.

\begin{tabular}{|c|c|c|c|}
\hline \multicolumn{4}{|c|}{ Table 1} \\
\hline & Unilateral & Bilateral & Total \\
\hline Failed follow up & 3 & 2 & 5 \\
\hline Repetition of $A A B R$ & 5 & 3 & 8 \\
\hline ABR/ASSR & 4 & 13 & 17 \\
\hline Hearing within normal limits & 2 & 1 & 3 \\
\hline slight hearing loss & - & 1 & 1 \\
\hline Mild hearing loss & - & 2 & 2 \\
\hline Moderate hearing loss & 1 & - & 1 \\
\hline Moderately severe hearing loss & - & 3 & 3 \\
\hline Severe hearing loss & 1 & 5 & 6 \\
\hline Profound hearing loss & - & 1 & 1 \\
\hline Total & 12 & 18 & 30 \\
\hline
\end{tabular}

\section{Discussion}

According to our results the sensitivity of AABR was $\mathbf{9 7 . 5 4 \%}$ and the specificity $\mathbf{8 7 . 5 \%}$ with a Positive Predictive Value 99.54\% and a Negative Predictive Value 56\%. The OAE's had sensitivity $82.92 \%$ and specificity $100 \%$ with a Positive Predictive Value $100 \%$ and a Negative Predictive Value $24 \%$. (Table 2), presenting AABR to have higher sensitivity whereas OAE's have higher specificity .

Consequently the combination of the two tests seem to be the most accurate for the earliest detection of sensoneural hearing loss.

\begin{tabular}{l|l|l|l|l|l|l|}
\multicolumn{7}{|c|}{ Table 2 } \\
\hline Methods & Sensitivity & $\mathbf{9 5 \% C I}$ & Specificity & $\mathbf{9 5 \% C I}$ & PPV & NPV \\
\hline AABR & $97.54 \%$ & $\begin{array}{l}95.65 \% \text { to } \\
98.77 \%\end{array}$ & $87.50 \%$ & $\begin{array}{l}61.65 \% \text { to } \\
98.45 \%\end{array}$ & $99.54 \%$ & $56 \%$ \\
\hline OAE's & $82.92 \%$ & $\begin{array}{l}79.10 \% \text { to } \\
86.30 \%\end{array}$ & $100.00 \%$ & $\begin{array}{l}85.75 \% \text { to } \\
100.00 \%\end{array}$ & $100.00 \%$ & $24 \%$ \\
\hline
\end{tabular}

